\title{
Star-Shaped Probability Weighting Functions and Overbidding in First-Price Auctions
}

\author{
Olivier Armantier* Nicolas Treich ${ }^{\dagger}$
}

March 2009

\begin{abstract}
There is a debate about whether risk aversion is the main source of overbidding in a first-price independent private values auction. As an alternative, we adopt a non-expected utility framework, and identify an interpretable property on the probability weighting function which always induces overbidding.
\end{abstract}

Keywords: Probability Weighting Function, Auctions, Overbidding, Non-expected Utility, Risk Aversion.

JEL Classification: C70, C92, D44, D81.

*Federal Reserve Bank of New York, Université de Montréal, CIRANO, and CIREQ.

${ }^{\dagger}$ Toulouse School of Economics, (LERNA-INRA), Aile J.-J. Laffont, 21 all. de Brienne, 31042 Toulouse, France. Email: ntreich@toulouse.inra.fr. 


\section{Introduction}

It has been repeatedly observed in experiments that subjects participating in first-price independent private values (FIPV) auctions tend to bid above the risk neutral Bayesian Nash equilibrium. This tendency to "overbid" has often been rationalized by risk aversion within the expected utility framework (Milgrom and Weber, 1982; Cox, Smith and Walker, 1985, 1988). This explanation, however, has been widely criticized. ${ }^{1}$ In particular, risk aversion is often viewed with skepticism due to the low financial incentives typically involved in laboratory experiments. Moreover, numerous individual decisionmaking experiments have suggested that subjects weight non-linearly probabilities when making risky choices (Camerer, 1995). It appears therefore natural to expect that agents may use a non-linear probability weighting function (PWF) in games, such as auctions, where probabilities are involved.

In this note, we identify a simple property on the PWF, the so-called "star-shaped" PWF, that always induces overbidding in FIPV auctions. As we shall see, this property is weaker than the convexity of the PWF, but stronger than a mere underweighting of probabilities. Interestingly, the "starshaped" property is relevant to characterize the notion of risk aversion in Rank-dependent expected utility (RDEU) models.

\section{The Model}

Risk preferences are characterized by the RDEU model, which is one of the best-known non-expected utility model involving a non-linear PWF. ${ }^{2}$ In the

\footnotetext{
${ }^{1}$ Among those criticisms, risk aversion cannot explain the observed persistent overbidding in second-price or third-price auctions. See the December 1992 issue of the American Economic Review for a sample of the debates around the causes of overbidding, and Kagel (1995) for an excellent literature survey.

${ }^{2}$ An early reference is Quiggin (1982). There exist several versions of the RDEU model (Chew, 1983; Luce and Fishburn, 1991; Tversky and Kahneman, 1992; Wakker and Tversky, 1993; Schmidt and Zank, 2001; Safra and Segal, 2001). The RDEU model is the only possible generalization of expected utility that is separable in probabilities and outcomes consistent with first-order stochastic dominance (Quiggin, 1993, p. 72). It can explain experimental puzzles such as the Allais paradox and the common ratio effect. The most common PWF consistent with choice data is such that small probabilities are inflated and large probabilities are deflated, giving rise to the common inverse S-shaped PWF (Tversky and Fox, 1995; Prelec, 1998; Gonzalez and Wu, 1999).
} 
case of a binary lottery, the RDEU model reduces to

$$
\Phi(p) u\left(w_{2}\right)+(1-\Phi(p)) u\left(w_{1}\right)
$$

where $p$ is the probability of the best of the two monetary outcomes $w_{2} \geq w_{1}$, and $\Phi$ is the PWF. As usual, we assume that $\Phi$ is differentiable and strictly increasing with $\Phi(0)=0$ and $\Phi(1)=1$. Note that when $u(w)=w$, the model essentially reduces to the dual theory of Yaari (1987). When $\Phi(p)=p$, we are back to the standard expected utility model.

The RDEU model enables one to distinguish between the notions of weak and strong risk aversion. An individual is weakly risk averse if and only if he always declines a random variable in favor of its expected value. An individual is strongly risk averse if and only if he always dislikes any meanpreserving spread of risk. Under expected utility, weak and strong risk aversion are equivalent to the concavity of $u$. Under RDEU, strong risk aversion is equivalent to $u$ concave and $\Phi$ convex (Chew, Karni and Safra, 1987), and weak risk aversion is equivalent to $u$ concave and $\Phi(p) \leq p$ for every $p \in[0,1]$ (Quiggin, 1993; Cohen, 1995).

We now introduce the FIPV auction model. $N \geq 2$ agents with identical risk preferences (i.e. identical $u$ and $\Phi$, as well as identical initial wealth $w)$ participate in an auction in which they each submit a sealed bid for an indivisible object. Agent $i=1, \ldots, N$ has a private value $v_{i}$ for the object. This private value is drawn independently from a distribution with cumulative $F($.$) , density f($.$) and support [\underline{v}, \bar{v}]$ where $\underline{v} \geq 0$. The highest bidder gets the object. His payoff is equal to his own valuation of the object minus his bid, $v_{i}-b_{i}$. The other bidders receive no payoffs.

We denote $p\left(b_{i}, B\right) \equiv P\left[b_{i}>B\left(v_{j}\right), \forall j \neq i\right]$ the probability that bidder $i$ wins the auction when she selects a bid $b_{i}$, while each of her opponents uses the bid function $B$. A strategy $B^{*}$ is then a symmetric Bayesian Nash equilibrium of this game if it satisfies the following optimization and fixed point problems,

$$
\begin{aligned}
B^{*}\left(v_{i}\right) & =\underset{\underline{v} \leq b_{i} \leq v_{i}}{\operatorname{ArgMax}} \Phi\left(p\left(b_{i}, B^{*}\right)\right) u\left(w+v_{i}-b_{i}\right)+\left(1-\Phi\left(p\left(b_{i}, B^{*}\right)\right)\right) u(w) \\
\forall v_{i} & \in[\underline{v}, \bar{v}] \text { and } \forall i=1, \ldots, N .
\end{aligned}
$$

In words, $B^{*}\left(v_{i}\right)$ is the bidder $i$ 's best-reply to other bidders' equilibrium strategy $B^{*}($.$) , given that all bidders have the same preferences. The only$ difference compared to the standard FIPV auction model is that the PWF $\Phi$ 
may be non-linear. If we restrict our attention to monotonic strategies, then the maximization in (1) is equivalent to maximizing over $b_{i}$

$$
\Phi\left(F\left(B^{*-1}\left(b_{i}\right)\right)^{N-1}\right) U\left(v_{i}-b_{i}\right)
$$

where $B^{*-1}($.$) stands for the inverse of B^{*}($.$) , and U$ is an indirect utility function defined by $U(x)=u(w+x)-u(w)$. Notice that $U$ is concave if and only if $u$ concave.

Differentiating with respect to $b_{i}$ and setting the obtained expression equal to zero gives the following differential equation

$$
B^{*^{\prime}}\left(v_{i}\right)=(N-1) f\left(v_{i}\right) \frac{\Phi^{\prime}\left(F\left(v_{i}\right)^{N-1}\right) F\left(v_{i}\right)^{N-2}}{\Phi\left(F\left(v_{i}\right)^{N-1}\right)} \frac{U\left(v_{i}-B^{*}\left(v_{i}\right)\right)}{U^{\prime}\left(v_{i}-B^{*}\left(v_{i}\right)\right)} \forall v_{i} \in[\underline{v}, \bar{v}] .
$$

Together with the boundary condition $B^{*}(\underline{v})=\underline{v}$, this differential equation characterizes a Bayesian symmetric Nash equilibrium bidding behavior in an auction where bidders have a PWF $\Phi($.$) .$

\section{The Effect of a Non-linear PWF}

\subsection{The Star-shaped PWF and the Main Result}

We introduce a specific class of PWF that will permit to sign the effect of a non-linear PWF on the equilibrium bidding strategy compared to a linear PWF. We coin this class the star-shaped PWF.

Definition Let a PWF $\Phi(p)$ with $\Phi(0)=0$ and $\Phi(1)=1$; then $\Phi(p)$ is star-shaped if and only if $\Phi(p) / p$ is increasing in $p$.

The term star-shaped is taken from Chateauneuf, Cohen and Meilijson (2004). ${ }^{3}$ A star-shaped PWF is useful to capture risk aversion toward one of their four specific types of increase in risk, that is, a right-monotone increase in risk. ${ }^{4}$ A star-shaped PWF implies that the chord to the PWF drawn

\footnotetext{
${ }^{3} \mathrm{~A}$ function $\Phi$ is star-shaped at $\mu$ if $(\Phi(x)-\Phi(\mu)) /(x-\mu)$ is non-decreasing in $x$. The exact term is that $\Phi$ is star-shaped at 0 . See also Landsberger and Meilijson (1990) where the term is applied to utility functions.

${ }^{4}$ The four types are respectively i) a mean-preserving increase in risk, ii) a monotone increase in risk, iii) a left-monotone increase in risk and iv) a right-monotone increase
} 
from 0 to $p$ must lay above $\Phi(p)$ for every $p$. This property is equivalent to assuming that the slope of the chord is lower than the slope of the tangent to $\Phi(p)$ at $p$, namely

$$
\Phi(p) / p \leq \Phi^{\prime}(p) \text { for every } p \in[0,1] .
$$

It is immediate that any convex PWF is star-shaped. Hence the notion of strong risk aversion as defined by Chew, Karni and Safra (1987) implies that, together with $u$ concave, the PWF is star-shaped in the RDEU model. Furthermore, observe that $\Phi$ star-shaped implies underweighting everywhere, i.e. $\Phi(p) \leq p$. Hence, assuming that $u$ is concave, a star-shaped PWF implies weak risk aversion.

We now compare $B^{*}(v)$, the symmetric equilibrium bidding strategy under a PWF $\Phi$ as given by the general condition (2), to the corresponding equilibrium condition in the expected utility case $(\Phi(p)=p)$ denoted $B_{0}^{*}(x)$. Assume that $\Phi$ is star-shaped, or equivalently that $\Phi^{\prime}(p) \geq \Phi(p) / p$ for every $p \in[0,1]$. Then, we get

$$
\begin{aligned}
B^{*^{\prime}}(v)-B_{0}^{*^{\prime}}(v)= & (N-1) f(v) F(v)^{N-2}\left[\frac{\Phi^{\prime}\left(F(v)^{N-1}\right)}{\Phi\left(F(v)^{N-1}\right)} \frac{U\left(v-B^{*}(v)\right)}{U^{\prime}\left(v-B^{*}(v)\right)}\right. \\
& \left.-\frac{1}{F(v)^{N-1}} \frac{U\left(v-B_{0}^{*}(v)\right)}{U^{\prime}\left(v-B_{0}^{*}(v)\right)}\right] \\
\geq & (N-1) \frac{f(v)}{F(v)}\left[\frac{U\left(v-B^{*}(v)\right)}{U^{\prime}\left(v-B^{*}(v)\right)}-\frac{U\left(v-B_{0}^{*}(v)\right)}{U^{\prime}\left(v-B_{0}^{*}(v)\right)}\right],
\end{aligned}
$$

by assumption and since $U, U^{\prime}, \Phi$ and $\Phi^{\prime}$ are positive.

From the last inequality, we have that, for any $v, B^{*}(v)=B_{0}^{*}(v)$ implies $B^{*^{\prime}}(v) \geq B_{0}^{*^{\prime}}(v)$. We thus have a single crossing property. This property means that the function $B^{*}($.$) can only cross the function B_{0}^{*}($.$) from below.$ Since $B^{*}(\underline{v})=B_{0}^{*}(\underline{v})=\underline{v}$, the function $B^{*}(v)$ is always be larger than $B_{0}^{*}(v)$ for any $v$ such that $v \geq \underline{v}$. This yields the following proposition.

in risk. In short, a right-monotone increase in risk corresponds to a risk spread in the domain of gains. To have an intuition for this, consider a lottery which yields either $x / p$ with probability $p>0$, or 0 otherwise. The expectation of this lottery is $x$. Notice that a decrease in $p$ corresponds to a specific mean-preserving spread in the domain of gains. Denote $C(p)$ the risk premium toward this lottery, defined by $u(w+x-C(p))=$ $\Phi(p) u(w+x / p)+(1-\Phi(p)) u(w)$; for a $u$ linear we thus have $C(p)=x(1-\Phi(p) / p)$. Hence, assuming a linear utility function, the risk premium increases in this particular type of mean-preserving spread in the domain of gains if and only if $\Phi$ is star-shaped. 
Proposition 1 Assume that participants in a first-price independent private values auction have identical risk preferences characterized by a Rankdependent expected utility model with a probability weighting function $\Phi$. The equilibrium bid is higher in an auction in which participants have a starshaped $\Phi$ compared to an auction in which they have a linear $\Phi$ (i.e., they are expected utility maximizers).

\subsection{A Closed-form Solution and Estimates of Risk Pref- erences Parameters}

Assume a power utility function and a zero initial wealth, so that $U(x)=$ $x^{1-r} /(1-r)$ with $r \in\left[0,1\left[. \quad\right.\right.$ Moreover, assume $\Phi(p)=p^{\beta}$, with $\beta \geq$ 1. Therefore, $\Phi$ is star-shaped since it is convex. Also assume a uniform distribution $F(v)=v$ over the support $[0,1]$. Under these assumptions, we derive a closed-form solution to the differential equation (2), which reduces to

$$
B^{*}(v)=\beta v(1+\beta-r)^{-1}
$$

for the two-bidder case. Observe that, compared to a linear PWF (i.e. $\beta=1$ ), a star-shaped PWF (i.e. $\beta \geq 1$ ) yields a higher equilibrium bid, consistent with Proposition 1. Note also that the equilibrium bid can increase indifferently with either $\beta$ the curvature of the PWF, or $r$ the constant relative risk aversion parameter.

This example also illustrates how our theoretical model captures recent empirical results. In particular, using experimental data, Goeree, Holt and Palfrey (2002) as well as Armantier and Treich (2007) obtain an estimated value for $r$ around 0.6 when they impose a linear PWF (i.e. $\beta=1),{ }^{5}$ while they obtain a quadratic PWF (i.e. $\beta \approx 2$ ) when assuming a linear utility (i.e. $r=0$ as in Yaari, 1987). When they estimate a model combining a

\footnotetext{
${ }^{5}$ The estimate of $r=0.6$ is consistent with results obtained under risk aversion with other experimental and non-experimental auction data (Harrison 1990; Cox and Oaxaca 1996; Chen and Plott 1998; Campo, Perrigne and Vuong 2001). This value for $r$ may be considered low compared to values obtained in the risky choice literature (e.g., in finance or insurance), usually larger than 1 , and often in the vicinity of 3 or 4 . This comparison to other risk aversion estimates is, however, somewhat misleading. Indeed, auction models have been typically estimated under the assumption that wealth $w$ is equal to 0 . This assumption is, obviously, not realistic and leads to a bias in favor of a low level of risk aversion. In fact, constant relative risk aversion estimates increase dramatically when positive levels of wealth are introduced.
} 
non-linear utility and a PWF, Armantier and Treich (2007) find a convex PWF and a concave utility function. However, when estimated on data from experienced subjects, both kinds of nonlinearities decline significantly. Moreover, Armantier and Treich (2007) find that their subjects are heterogeneous with respect to risk preferences and to the PWF.

\section{Conclusion}

We have shown in this note that a star-shaped PWF could rationalize overbidding in FIPV auctions. We conclude with three observations. First, this paper is one of the first attempts at explaining a puzzle in a strategic game using the RDEU model. Second, our result is not inconsistent with the general idea that "risk aversion" is still the main determinant of overbidding; indeed the star-shaped PWF property is relevant to characterize a broader notion of risk aversion that has been proposed in RDEU models (Chew, Karni and Safra, 1987; Quiggin, 1993; Chateauneuf, Cohen and Meilijson, 2004). Third, our result is consistent with the recent PWF estimates from the FIPV auction experiments of Goeree, Holt and Palfrey (2002) and of Armantier and Treich (2007). Nevertheless, our result only applies to the case where bidders are identical, and therefore cannot fully account for possible heterogeneity in risk preferences and in the PWF. 


\section{References}

Armantier O. and N. Treich, 2007, "Subjective Probabilities in Games: An Application to the Overbidding Puzzle," International Economic Review, forthcoming.

Camerer C., 1995, "Individual Decision Making," In: Kagel, J.H. \& Roth, A.E. (eds.): Handbook of Experimental Economics, Princeton, NJ: Princeton University Press, 587-703.

Campo S., Perrigne I. and Q. Vuong, 2001, "Asymmetry in First-Price Auctions with Affiliated Private Values," Journal of Applied Econometrics, 2, 179-207.

Chateauneuf, A., Cohen M. and I. Meilijson, 2004, "Four Notions of Mean Preserving Increase in Risk, Risk Attitudes and Applications to the RankDependent Expected Utility model," Journal of Mathematical Economics, 40, 547-71.

Chen K.Y. and C. Plott, 1998, "Nonlinear Behavior in Sealed Bid FirstPrice Auctions," Games and Economic Behavior, 25, 34-78.

Chew S.H., 1983, "A Generalization of the Quasilinear Mean with an Applications to the Measurement of Income Inequality and Decision Theory Resolving the Allais Paradox," Econometrica, 51, 1065-92.

Chew C.S., Karni E. and Z. Safra, 1987, "Risk-Aversion in the Theory of Expected Utility with Rank Dependent Probabilities," Journal of Economic Theory, 42, 370-381.

Cohen M.D., 1995, "Risk-aversion Concepts in Expected- and Non-expectedutility Models," The Geneva Papers on Risk and Insurance Theory, 20, 73-91.

Cox J. and R. Oaxaca, 1996, "Is Bidding Behavior Consistent with Bidding Theory for Private Value Auctions," in R. M. Isaac, ed., Research in Experimental Economics, Vol. 6, Greenwich, Conn.: JAI Press, 131-148.

Cox J., Smith V. and J. Walker, 1985, "Experimental Development of Sealed-Bid Auction Theory: Calibrating Controls for Risk Aversion," American Economic Review Papers and Proceedings, 75, 160-165.

Cox J., Smith V. and J. Walker, 1988, "Theory and Individual Behavior of First-Price Auctions," Journal of Risk and Uncertainty, 1, 61-99.

Goeree J., Holt C., and T. Palfrey, 2002, "Quantal Response Equilibrium and Overbidding in Private-value Auctions," Journal of Economic Theory, $104,247-272$. 
Gonzalez R. and G. Wu, 1999, "On the Shape of the Probability Weighting Function," Cognitive Psychology, 38, 129-66.

Harrison G.W., 1990, "Risk Attitudes in First-price Auction Experiments: A Bayesian Analysis," Review of Economics and Statistics, 72, 541-546.

Kagel J., 1995, "Auctions: A Survey of Experimental Research," In: Kagel, J.H. \& Roth, A.E. (eds.): Handbook of Experimental Economics, Princeton, NJ: Princeton University Press, 501-586.

Landsberger M. and I. Meilijson, 1990, "Lotteries, Insurance and Starshaped Utility Functions," Journal of Economic Theory, 52, 1-17.

Luce R.D. and P.C. Fishburn, 1991, "Rank- and Sign-dependent Linear Utility Models for Finite Order Gambles," Journal of Risk and Uncertainty, 4, 25-59.

Milgrom P. and R. Weber, 1982, "A Theory of Auctions and Competitive Biddings," Econometrica, 50, 1089-1122.

Prelec D., 1998, "The Probability Weighting Function," Econometrica, $66: 3,497-527$.

Quiggin J., 1982, "A Theory of Anticipated Utility," Journal of Economic Behavior and Organization, 3, 323-43.

Quiggin J., 1993, Generalized Expected Utility Theory, Kluwer Academic Publishers.

Safra Z. and U. Segal, 2001, "Rank-dependent Preferences without Ranking Axioms," Journal of Mathematical Economics, 35, 547-62.

Schmidt U. and H. Zank, 2001, "A New Axiomatization of Rank-dependent Expected Utility with Tradeoff Consistency for Equally Likely Outcomes," Journal of Mathematical Economics, 35, 483-91.

Tversky A. and C.R. Fox, 1995, "Weighting Risk and Uncertainty," Psychological Review, 102, 269-83.

Tversky A. and D. Kahneman, 1992, "Cumulative Prospect Theory: An Analysis of Decision under Uncertainty," Journal of Risk and Uncertainty, 5, 297-323.

Wakker P.P. and A. Tversky, 1993, "An Axiomatization of Cumulative Prospect Theory," Journal of Risk and Uncertainty, 7, 147-76.

Yaari M.E., 1987, "The Dual Theory of Choice Under Risk," Econometrica, 55, 95-115. 